\title{
Enhanced spin Hall effect of light by transmission in a polymer
}

Takayama, Osamu; Puentes, Graciana

Published in:

Optics Letters

Link to article, DOI:

10.1364/OL.43.001343

Publication date:

2018

Document Version

Peer reviewed version

Link back to DTU Orbit

Citation (APA):

Takayama, O., \& Puentes, G. (2018). Enhanced spin Hall effect of light by transmission in a polymer. Optics Letters, 43(6), 1343-1346 . https://doi.org/10.1364/OL.43.001343

\section{General rights}

Copyright and moral rights for the publications made accessible in the public portal are retained by the authors and/or other copyright owners and it is a condition of accessing publications that users recognise and abide by the legal requirements associated with these rights.

- Users may download and print one copy of any publication from the public portal for the purpose of private study or research.

- You may not further distribute the material or use it for any profit-making activity or commercial gain

- You may freely distribute the URL identifying the publication in the public portal

If you believe that this document breaches copyright please contact us providing details, and we will remove access to the work immediately and investigate your claim 


\title{
Enhanced spin Hall effect of light by transmission in a polymer
}

\author{
Osamu Takayama ${ }^{1}$ and Graciana Puentes ${ }^{2,3}$ \\ ${ }^{1}$ Department of Photonics Engineering, Technical University of Denmark, Ørsteds Plads, Building 343, DK-2800 Kgs. Lyngby, Denmark \\ ${ }^{2}$ Universidad de Buenos Aires. Facultad de Ciencias Exactas y Naturales. Departamento de Física. Buenos Aires, Argentina. \\ ${ }^{3}$ CONICET-Universidad de Buenos Aires. Instituto de Física de Buenos Aires (IFIBA). Buenos Aires, Argentina. \\ ${ }^{*}$ Corresponding authors: gracianapuentes@gmail.com, otak@fotonik.dtu.dk
}

We demonstrate experimentally the lateral circular birefringence of a tunable birefringent polymer, the first example of the spin Hall effect of light in a polymeric material, and we demonstrate that this light shift can be significantly enhanced by tuning the effective birefringence in the polymer. We report experimental observations of this effect using polarimetric techniques and quantum-weak-measurement techniques, reporting a weak amplification factor of 200. () 2018 Optical Society of America

OCIS codes: (260.0260) Physical optics; (260.5430) Polarization; (260.1440) Birefringence.

http://dx.doi.org/10.1364/ao.XX.XXXXXX

Spin-Orbit Interactions (SOI) of light refer to the coupling of different internal degrees of freedom of the radiation field, such as polarization and spatial degrees of freedom, as a result of propagation of light in different media [1]. A remarkable example of SOI of light, the spin Hall effect of light or photonic spin Hall effect, has recently attracted attention in a number of fast growing fields, ranging from photonics, plasmonics, nanooptics and quantum optics to metamaterials [2]. Most SOI effects originate from space-variant geometric phases and result in spindependent redistribution of light intensity [2]. When the system has cylindrical symmetry, SOIs generate a spin-dependent vortex in the z-propagating light [2-15]. If the cylindrical symmetry is broken, SOIs bring about the spin-Hall effect of light, i.e., a spin-dependent transverse y-shift of light intensity [1321]. An example of the latter effect is the so-called transverse Imbert-Fedorov (IF) beam shift which occurs when a paraxial optical beam is reflected or refracted at a plane interface [19-21].

Up to present, the spin Hall effect of light has been observed for various material and geometrical settings, such as glass [22], gratings [4, 10], liquid crystals [5], dielectric spheres [11], metal [23] and magnetic [24] films, uniaxial birefringent crystals [25], graphene [26]. Furthermore, recently plasmonic [27] and dielectric [28] meta-surfaces [29], as well as by hyperbolic metamaterials [30], have been intensively studied due to their ability to manipulate polarization states by the design of artificial surface structures in subwavelength scale. Photonic spin-Hall effect offers device applications ranging from spin-dependent beam
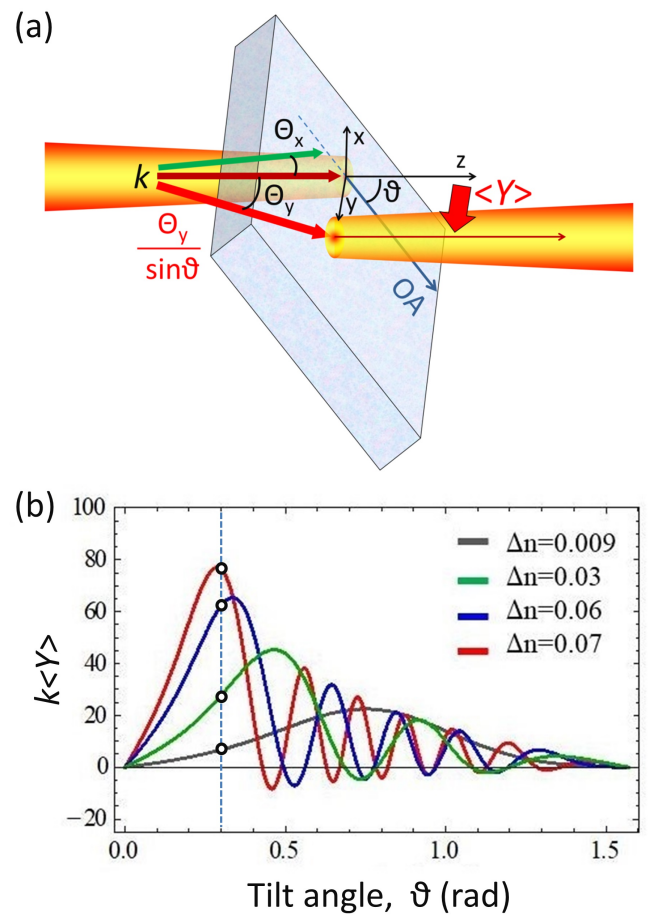

Fig. 1. (a) 3D geometry illustrating transmission of a paraxial beam through a tilted transparent polymer film. The beam experiences nano-meter scale transverse shift $\langle Y\rangle$ due to spin Hall effect of light. The paraxial angles $\left(\Theta_{y}, \Theta_{x}\right)$ determine the direction of propagation of the wave vectors $k$ in the incident beam. The transverse $\Theta_{y}$ deflections of the wave vectors rotate the planes of wave propagation with respect to the anisotropy axis by the angle $\Theta_{y} / \sin (\vartheta)$. (b) Numerical simulation displaying enhanced spin Hall effect of light by transmission in a tunable polymer of $50 \mu \mathrm{m}$ thickness with different birefringence: $\Delta n=0.009$ (Grey, index difference in Quartz), $\Delta n=0.03$ (Green), $\Delta n=0.06$ (Blue), and $\Delta n=0.07$ (Red, index difference in stretched polymers [34]). Note that the vertical dashed line at $\vartheta=0.3$ (rad) is to show the tunability of beam shift by different birefringence. 
splitters [29] to surface sensors [31].

In this letter, we experimentally demonstrate significant enhancement of the spin-Hall effect of light by transmission through a $50 \mu \mathrm{m}$ thick birefringent polymer film, as illustrated in Fig. 1(a). The enhancement in polymer films can be orders of magnitude larger compared with a Quartz crystal plate of the same thickness. Moreover, the enhancement can be tuned by increasing or decreasing the effective birefringence in the polymer. Furthermore, to the best of our knowledge, this is the first time the spin Hall effect of light in a polymeric material is reported. Polymers are widely used as optical materials from transmission media to light sources with the advantages of low processing cost, mechanical flexibility, ease of large area fabrication and so on [32]. Moreover, birefringent polymers are typically produced by electronic modulation, such as liquid crystals [33] or by stress induced mechanical effects [34]. Therefore, this provides an external means of control for the birefringence induced in the polymer and could in turn be used as an optical switch in the nano-meter range, as opposed to natural crystals or metal where the birefringence if fixed by the parameters of the media.

The complete theory for light transverse shifts in uniaxial crystals was described in [25]. For an input state described by a Jones vector $|\psi\rangle$ and a transmitted state described by Jones vector $\left|\psi^{\prime}\right\rangle$, the anisotropic transverse shift or spin-Hall effect of light is given by the expectation value of the position operator:

$$
\langle Y\rangle=\left\langle\psi^{\prime}|\hat{Y}| \psi^{\prime}\right\rangle=\frac{\cot (\vartheta)}{k}\left[-\sigma\left(1-\cos \left(\phi_{0}\right)\right)+\chi \sin \left(\phi_{0}\right)\right],
$$

where $\phi_{0}$ represent the phase difference between ordinary and extraordinary wave propagating through the birefringent medium, and $(\sigma, \chi)$,represent the Stokes parameters for light, which quantify the degree of polarization in the diagonal and circular basis, respectively.

The spin Hall effect can be measured either directly, via subwavelength shift [Eq. (1)] of the beam centroid [19, 20, 35], or via various other methods including quantum weak measurements [21, 36-41]. The latter method allows for significant amplification of the shift using almost crossed polarizers at the input and output of the system, respectively. As before, the input polarizer corresponds to a pre-selected state $|\psi\rangle=(\alpha, \beta)^{T}$ (where $T$ stands for transpose operation), while the output polarizer corresponds to another post-selected polarization state $\left|\psi^{\prime}\right\rangle=\left(\alpha^{\prime}, \beta^{\prime}\right)^{T}$. The resulting beam shifts are determined by the weak value $\langle Y\rangle_{\text {weak }}$, instead of expectation values $\langle Y\rangle$, which can exhibit quantum weak amplification effect and lay outside the bounds of the spectrum of the operator. We analyze quantum weak amplification of the spin-Hall shift, considering an initial beam with $e$ polarization $|\psi\rangle=(1,0)^{T}$, while the post-selection polarizer is nearly orthogonal $|\phi\rangle=(\epsilon, 1)^{T},|\epsilon|<<1$. The weak value yields:

$$
\langle Y\rangle_{\text {weak }}=\frac{1}{\epsilon k} \sin \left(\phi_{0}\right) \cot (\vartheta)+\frac{z}{z_{R}} \frac{1}{\epsilon k}\left(1-\cot \left(\phi_{0}\right)\right) \cot (\vartheta),
$$

where $z_{R}$ is the Rayleigh length. The second (angular) term, becomes dominant in the far field zone, and presents weak amplification due to two reasons. Firstly, because $|\epsilon|<<1$, and secondly because $z>>z_{R}$. Note that the maximal achievable weak amplification at $|\epsilon| \approx\left(k \omega_{0}\right)^{-1}$ is of the order of the beam waist $\omega_{0} z / z_{R}$.

To show the tunability of the photonic spin Hall shift by tuning the birefringence of polymer film, we calculate the expectation value of the centroid displacement for a birefringent polymer, modeled as a tilted uniaxial plate of thickness $d=50$ $\mu \mathrm{m}$ with various birefringence as shown in Fig. 1(b). Here we assume that the birefringence of polymer film can be tuned by stretching [34]. For a given tilt angle of $\vartheta=0.3$ [rad] (dashed line if Fig. $1(\mathrm{~b}))$, the beam shift can be tuned from to $k\langle Y\rangle$ $=15\left(\Delta_{n}=0.009\right.$, Grey) to $k<Y>=77\left(\Delta_{n}=0.07\right.$, Red $)$. This tuning of beam shift by varying the birefringence of polymer in a dynamic manner may lead to optical switches. Furthermore, we can observe the general tendency of increasing beam shift for larger anisotropy of polymer film.

The phase difference can be expressed as:

$$
\phi_{0}(\vartheta)=k\left[n_{o} d_{o}(\vartheta)-\bar{n}_{e}(\vartheta) d_{e}(\vartheta)\right] .
$$

Here, $n_{0}=1.54$ is the refractive index for the ordinary wave, $n_{e}(\vartheta)=n_{o} n_{e} / \sqrt{n_{e}^{2} \cos (\vartheta)^{2}+n_{o}^{2} \sin (\vartheta)^{2}}$ is the refractive index for the extraordinary wave propagating at the angle $\vartheta$ to the optical axis, $\bar{n}_{e}=n_{e}(\pi / 2)=1.60$, and the distances of propagation of the ordinary and extraordinary rays in the tilted plate are:

$$
d_{e}(\vartheta)=\frac{\bar{n}_{e}(\vartheta) d}{\sqrt{\bar{n}_{e}^{2}(\vartheta)-\cos (\vartheta)^{2}}}, \quad d_{o}(\vartheta)=\frac{n_{o} d}{\sqrt{n_{o}^{2}-\cos (\vartheta)^{2}}} .
$$
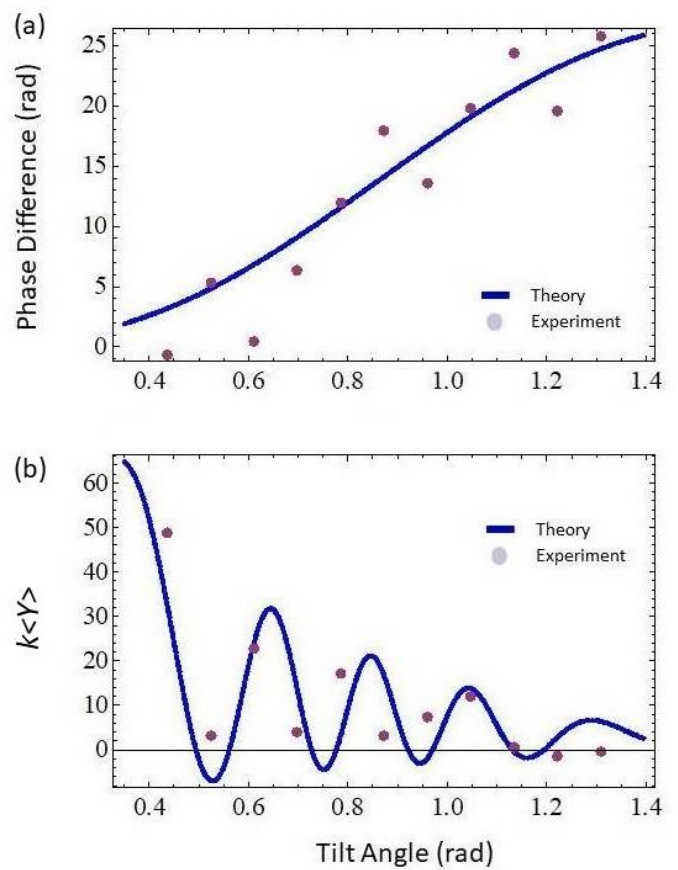

Fig. 2. Polarimetric measurement of (a) Phase difference $\Phi_{0}$ between ordinary and extraordinary polarizations, (b) adimensional transverse spin Hall shift $(k\langle Y\rangle)$, with wave-number $k=\frac{2 \pi}{\lambda}$. Theoretical prediction is indicated with blue curve and experimental data is indicated with purple dots. Agreement between experiment and theory is apparent.

Using Eqs. (1)-(3), we plot the phase difference and spin-Hall shift (blue curves) as functions of the tilt angle $\vartheta$ as shown in in Figs. 2(a) and 2(b). One can see that the transverse beam shift $\langle Y\rangle$ due to the spin-Hall effect reaches wave-length order magnitude. We note that in previous works beam shifts on the order of the wave-length were only reported indirectly, via 


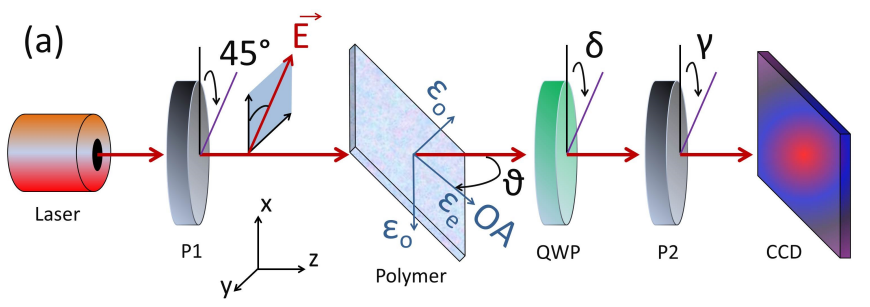

(b)

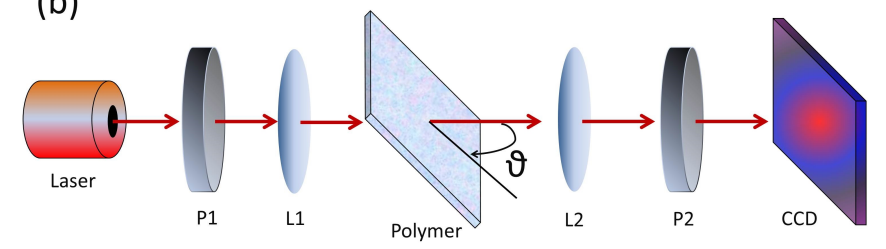

Fig. 3. Schematic illustration of experimental setups for (a) polarimetric and (b) quantum weak measurements. P1 and P2 represent double Glan-Laser polarizers (Thorlabs GL10), QWP is quarter wave plate. Lenses are denoted as L1 and L2. Laser is He-Ne (Melles Griot Griot 05-LHR-111) laser with the emission wavelength of $633 \mathrm{~nm}$, the CCD camera model is Thorlabs WFS150-5C. The ordinary and extraordinary permitivities of the polymer film and their axes are denoted as $\varepsilon_{0}$ and $\varepsilon_{e}$, respectively.

quantum-weak-amplification techniques [21, 23, 25], a feature which further differentiates our work from former results. In contrast to the IF shift in the reflection/refraction problems, here the transverse shift as a function of $v$ displays two-scale behavior. Namely, the fast oscillations in Fig. 2(b) originate from the term $\left(1-\cos \left(\Phi_{0}\right)\right)$, whereas the slow envelope corresponds to the universal $\cot (\vartheta)$ factor in SOI terms.

To verify the above theoretical predictions, we performed a series of experimental measurements using the setups shown in Fig. 3. We use a sample of free-standing birefringent polymer foil, similar to the type Newport 05RP32-1064. As a source of incident Gaussian beam, we employed a He-Ne laser (Melles Griot Griot 05-LHR-111) of wavelength $\lambda=633 \mathrm{~nm}$. The laser radiation was collimated using a microscope objective lens. We measure the anisotropic phase difference $\Phi_{0}$ versus the angle of the tilt $v$ via Stokes polarimetry methods [42]. For this purpose we used the setup shown in Fig. 3(a). The double Glan-Laser polarizer (Thorlabs GL10) (P1) selected the desired linear-polarization state in the incident beam. In the first experiment, this was $45^{\circ}$ polarization, i.e., $\alpha=\beta=1 / \sqrt{2}$. The beam then propagates through the polymer, and the Stokes parameters are measured using a Quarter Wave-Plate (QWP) at a retardation angle $\delta$, and a second polarizer P2, with angle $\gamma$, as indicated in Fig. 3(a). The phase difference can be obtained via the Stokes parameters using the expression:

$$
\phi_{0}=\tan ^{-1}\left(\frac{S_{3}}{S_{2}}\right),
$$

where $S_{3}=I\left(90^{\circ}, 45^{\circ}\right)-I\left(90^{\circ}, 135^{\circ}\right)$ is the normalized Stokes parameter for circular polarization, and $S_{2}=I\left(0^{\circ}, 45^{\circ}\right)-$ $I\left(0^{\circ}, 135^{\circ}\right)$ is the normalized Stokes parameter in the diagonal basis, where the normalization factor $S_{0}$ is given by the total intensity of the beam. The measured phase using Eq. (5) is wrapped in the range $(-\pi, \pi)$. In order to determine the unwrapped phase difference we use an unwrapping algorithm [25], with a tolerance set to 0.001 radians. The measured unwrapped phase difference is displayed in Fig. 3(a) (purple dots). We note the reduced agreement between theory and experiment, as compared with Ref.[25]. We ascribe this difference to the fact that the theoretical model for the phase difference (Eq. 3) is exact for a unaxial crystal, however it is only approximate for a polymer film. The experimental spin Hall effect using Stokes polarimetry is shown in Fig. 2(b) (purple dots). where $S_{3}=I\left(90^{\circ}, 45^{\circ}\right)-I\left(90^{\circ}, 135^{\circ}\right)$ is the normalized Stokes parameter for circular polarization, and $S_{2}=I\left(0^{\circ}, 45^{\circ}\right)-I\left(0^{\circ}, 135^{\circ}\right)$ is the normalized Stokes parameter in the diagonal basis, where the normalization factor $S_{0}$ is given by the total intensity of the beam. The measured phase using Eq. (5) is wrapped in the range $(-\pi, \pi)$. In order to determine the unwrapped phase difference we use an unwrapping algorithm [25], with a tolerance set to 0.001 radians. The measured unwrapped phase difference is displayed in Fig. 2(a) (purple dots). The experimental spin Hall effect using Stokes polarimetry is shown in Fig. 2(b) (purple dots). The agreement between experiment and theory is

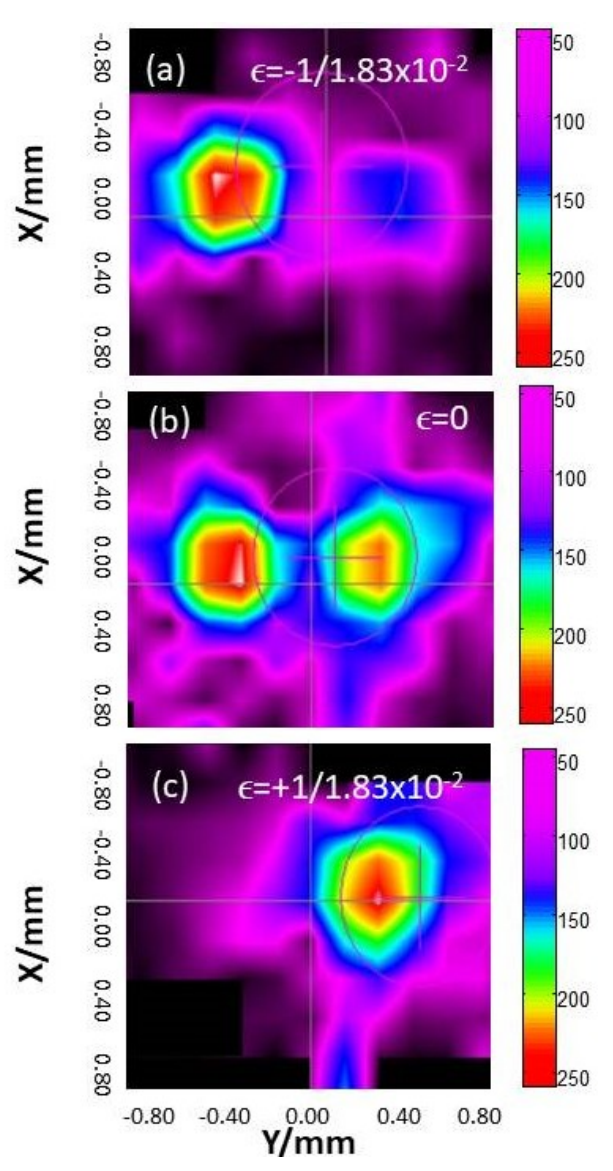

Fig. 4. Transverse intensity distributions (a.u.) for an opolarized beam transmitted through a tilted polymer film and post-selected in the almost e-polarized state, for a tilt angle $\vartheta=20^{\circ}$. (a) Post-selected polarization state with $\epsilon=-1 / 1.83 \times 10^{-2}$. The beam centroid is shifted, corresponding to a weak value measurement of $\langle Y\rangle_{\text {weak }}=-500 \mu \mathrm{m}$. (b) With crossed polarizers $(\epsilon=0)$, the input Gaussian beam is split into a Hermite-Gaussian distribution. (c) Post-selected polarization state with $\epsilon=1 / 1.83 \times 10^{-2}$, corresponding to a weak value measurement of $\langle Y\rangle_{\text {weak }}=+500 \mu \mathrm{m}$. Image in false color scale obtained with a CCD camera. 
apparent. We note the reduced agreement between theory and experiment, as compared with Ref.[25]. We ascribe this difference to the fact that the theoretical model for the phase difference (Eq. 3) is exact for a uniaxial crystal, but it is only approximate for a polymer film. We note that we observe a spin-Hall effect via Stokes polarimetry $(k<Y>)$ using a $50 \mu \mathrm{m}$ polymer film which is 10 times larger than the shift observed in Ref. [25], for a $1000 \mu \mathrm{m}$ Quartz sample. We ascribe this increase to the larger effective birefringence in the polymers [34], as demonstrated in the numerical simulations displayed in Figure 1(b).

Next, we performed the weak measurement of spin Hall shift, and observed quantum weak amplification effect using the quantum weak measurement setup in Fig. 3(b). The beam is imaged using a CCD camera (Thorlabs WFS150-5C). To this end we inserted two lenses (L1) and (L2) of focal distance $f=6$ $\mathrm{cm}$. Polarizers Glan-Laser Polarizers P1 and P2 produce the pre-selected and post-selected states, with polarization states $|\phi\rangle$ and $|\psi\rangle$, respectively. The first lens (L1), of focal length $6 \mathrm{~cm}$, produced a Gaussian beam with waist $\omega_{0}=30 \mu \mathrm{m}$, and a Rayleigh range $z_{R}=4.6 \mathrm{~mm}$. Therefore, for a CCD camera located at a distance $z=5 \mathrm{~cm}$ the propagation amplification factor results $z / z_{R}=10.86$. The amplification factor due to crossed polarizers results $1 / \epsilon \approx 1.83 \times 10^{2}$. For $k=\frac{2 \pi}{\lambda}$, the overall weak amplification factor becomes $A=\frac{z}{z_{R}} \times \frac{1}{k \epsilon}=200$, this is confirmed in the experiment where a displacement between centroids of $\Delta Y=1000 \mu \mathrm{m}$ between post-selection polarizers oriented at $\epsilon=-1 / 1.83 \times 10^{-2}$ [Fig. 4(a)], and $\epsilon=+1 / 1.83 \times 10^{-2}$ [Fig. 4(c)] is measured at a tilt angle $\vartheta=20^{\circ}$, thus amplifying the spin Hall effect by a factor $A=200$. For crossed polarizers $(\epsilon=0)$, the input Gaussian beam is split into a Hermite-Gaussian distribution [Fig. 4(b)], and the separation of the two peaks is approximately $\Delta Y=1000 \mu \mathrm{m}$ in $\langle Y\rangle$.

In conclusion, we experimentally demonstrated the enhanced lateral circular birefringence of a tilted birefringent polymer as the first example of the spin Hall effect of light in a polymer material. We reported experimental observations of this nano-meter scale effect using Stokes polarimetry techniques and quantumweak-measurement techniques, reporting a quantum weak amplification factor of 200. The birefringence in the polymer can be tuned using mechanical stress in the case of stress-induced birefringence or voltage in the case liquid crystals. Therefore this lateral shift could be used as an optical switch in the nano-meter scale, opening the doors to a myriad of novel applications in photonics, nano-optics, quantum optics, and metamaterials.

Funding. Agencia Nacional de Promocion Cientifica y Tecnologica, PICT2015-0710 Startup, UBACyT PDE 2016 and UBACYT PDE 2017, Argentina; Villum Fonden DarkSILD project, Denmark (11116); Direktør Ib Henriksens Fond, Denmark.

Acknowledgement. The authors are grateful to Konstantin Bliokh and Ricardo Depine for insightful discussion.

\section{REFERENCES}

1. X. Ling, X. Zhou, K. Huang, Y. Liu, C.-W. Qiu, H. Luo, and S. Wen, Rep. Prog. Phys. 80, 066401 (2017).

2. K. Y. Bliokh, F. J. Rodríguez-Fortuño, F. Nori, and A. V. Zayats, Nat. Photonics 9, 796 (2015).

3. F. Cardano and L. Marrucci, Nat. Photonics 9, 776 (2015).

4. G. Biener, A. Niv, V. Kleiner, and E. Hasman, Opt. Lett. 27, 1875 (2002).

5. L. Marrucci, C. Manzo, and D. Paparo, Phys. Rev. Lett. 96, 163905 (2006).

6. C. Schwartz and A. Dogariu, Opt. Express 14, 8425 (2006).
7. Z. Bomzon and M. Gu, Opt. Lett. 32, 3017 (2007).

8. Y. Zhao, J. S. Edgar, G. D. M. Jeffries, D. McGloin, and D. T. Chiu, Phys. Rev. Lett. 99, 073901 (2007).

9. T. A. Nieminen, A. B. Stilgoe, N. R. Heckenberg, and H. RubinszteinDunlop, J. Opt. A 10, 115005 (2008).

10. Y. Gorodetski, A. Niv, V. Kleiner, and E. Hasman, Phys. Rev. Lett. 101, 043903 (2008).

11. D. Haefner, S. Sukhov, and A. Dogariu, Phys. Rev. Lett. 102, 123903 (2009).

12. E. Brasselet, N. Murazawa, H. Misawa, and S. Juodkazis, Phys. Rev. Lett. 103, 103903 (2009).

13. K. Y. Bliokh, M. A. Alonso, E. A. Ostrovskaya, and A. Aiello, Phys. Rev. A 82, 063825 (2010).

14. O. G. Rodríguez-Herrera, D. Lara, K. Y. Bliokh, E. A. Ostrovskaya, and C. Dainty, Phys. Rev. Lett. 104, 253601 (2010).

15. K. Y. Bliokh, E. A. Ostrovskaya, M. A. Alonso, O. G. Rodríguez-Herrera, D. Lara, and C. Dainty, Opt. Express 19, 26132 (2011).

16. N. B. Baranova, A. Y. Savchenko, and B. Y. Zel'dovich, J. Exp. Thoer. Phys. Lett. 59, 232 (1994).

17. B. Y. Zel'dovich, N. D. Kundikova, and L. F. Rogacheva, J. Exp. Theor. Phys. Lett. 59, 766 (1994).

18. Z. Bomzon, G. Biener, V. Kleiner, and E. Hasman, Opt. Lett. 27, 1141 (2002).

19. A. Aiello and J. P. Woerdman, Opt. Lett. 33, 1437 (2008).

20. K. Y. Bliokh and A. Aiello, J. Opt. 15, 014001 (2013).

21. O. Hosten and P. Kwiat, Science 319, 787 (2008).

22. X. Zhou and X. Ling, Opt. Express 24, 3025 (2016).

23. X. Zhou, Z. Xiao, H. Luo, and S. Wen, Phys Rev. A 85, 043809 (2012).

24. J. Ren, Y. Li, Y. Qin, R. Wu, J. Yang, Y.-F. Xiao, H. Yang, and Q. Gong, Phys. Lett. 101, 171103 (2012).

25. K. Bliokh, C. Samlan, C. Prajapati,G. Puentes, N. Viswanathan, F. Nori, Optica 3, 1039 (2016).

26. B. Z. Rameshti and M. Zareyana, Appl. Phys. Lett. 103, 132409 (2013).

27. X. Yin, Z. Ye, J. Rho, Y. Wang, and X. Zhang, Science 339, 1405 (2013).

28. Ling, X. Zhou, X. Yi, W. shu, Y. Liu, S. Chen, H. Luo, S. Wen, and D. Fan, Light: Science \& Applications 4, e290 (2015).

29. Y. Liu, Y. Ke, H. Luo, and S. Wen, Nanophotonics 6, 51 (2017).

30. P. V. Kapitanova, P. Ginzburg, F. J. Rodríguez-Fortuño, D. S. Filonov, P. M. Voroshilov, P. A. Belov, A. N. Poddubny, Y. S. Kivshar, G. A. Wurtz, and A. V. Zayats, Nat. Commun. 5, 3226 (2014).

31. X. Zhou, X. Ling, H. Luoa, and S. Wen, Appl. Phys. Lett. 101, 251602 (2012).

32. J. Clark and G. Lanzani, Nat. Photon. 4, 438 (2010).

33. D.-K. Yang and S.-T. Wu, Fundamentals of Liquid Crystal Devices, 2nd ed. (John Wiley \& Sons, 2015).

34. J.-G. Lim, K. Kwak, and J.-K. Song, Opt. Express 25, 16409 (2017).

35. A. Ciattoni, G. Cincotti, and C. Palma, Phys. Rev. E 67, 036618 (2003).

36. Y. Aharonov, D. Z. Albert, and L. Vaidman, Phys. Rev. Lett. 60, 1351 (1988).

37. I. M. Duck, P. M. Stevenson, and E. C. G. Sudarshan, Phys. Rev. D 40, 2112 (1989).

38. A. G. Kofman, S. Ashhab, and F. Nori, Phys. Rep. 520, 43 (2012).

39. J. Dressel, M. Malik, F. M. Miatto, A. N. Jordan, and R. W. Boyd, Rev. Mod. Phys. 86, 307 (2014).

40. N. W. M. Ritchie, J. G. Story, and R. G. Hulet, Phys. Rev. Lett. 66, 1107 (1991).

41. G. Puentes, N. Hermosa, J. P. Torres, Phys Rev. Lett. 109, 040401 (2012).

42. C. Prajapati, S. Pidishety, and N. K. Viswanathan, Opt. Lett. 39, 4388 (2014). 


\section{REFERENCES}

1. X. Ling, X. Zhou, K. Huang, Y. Liu, C.-W. Qiu, H. Luo, and S. Wen, "Recent advances in the spin Hall effect of light," Rep. Prog. Phys. 80, 066401 (2017).

2. K. Y. Bliokh, F. J. Rodríguez-Fortuño, F. Nori, and A. V. Zayats, "Spinorbit interactions of light," Nat. Photonics 9, 796-808 (2015).

3. F. Cardano and L. Marrucci, "Spin-orbit photonics," Nat. Photonics 9 , 776-778 (2015).

4. G. Biener, A. Niv, V. Kleiner, and E. Hasman, "Formation of helical beams by use of Pancharatnam-Berry phase optical elements," Opt. Lett. 27, 1875-1877 (2002).

5. L. Marrucci, C. Manzo, and D. Paparo, "Optical spin-to-orbital angular momentum conversion in inhomogeneous anisotropic media," Phys. Rev. Lett. 96, 163905 (2006).

6. C. Schwartz and A. Dogariu, "Conservation of angular momentum of light in single scattering," Opt. Express 14, 8425-8433 (2006).

7. Z. Bomzon and M. Gu, "Space-variant geometrical phases in focused cylindrical light beams," Opt. Lett. 32, 3017-3019 (2007).

8. Y. Zhao, J. S. Edgar, G. D. M. Jeffries, D. McGloin, and D. T. Chiu, "Spinto-orbital angular momentum conversion in a strongly focused optical beam," Phys. Rev. Lett. 99, 073901 (2007).

9. T. A. Nieminen, A. B. Stilgoe, N. R. Heckenberg, and H. RubinszteinDunlop, "Angular momentum of a strongly focused Gaussian beam," J. Opt. A 10, 115005 (2008).

10. Y. Gorodetski, A. Niv, V. Kleiner, and E. Hasman, "Observation of the spin-based plasmonic effect in nanoscale structures," Phys. Rev. Lett. 101, 043903 (2008).

11. D. Haefner, S. Sukhov, and A. Dogariu, "Spin Hall effect of light in spherical geometry," Phys. Rev. Lett. 102, 123903 (2009).

12. E. Brasselet, N. Murazawa, H. Misawa, and S. Juodkazis, "Optical vortices from liquid crystal droplets," Phys. Rev. Lett. 103, 103903 (2009).

13. K. Y. Bliokh, M. A. Alonso, E. A. Ostrovskaya, and A. Aiello, "Angular momenta and spin-orbit interaction of nonparaxial light in free space," Phys. Rev. A 82, 063825 (2010).

14. O. G. Rodríguez-Herrera, D. Lara, K. Y. Bliokh, E. A. Ostrovskaya, and C. Dainty, "Optical nanoprobing via spin-orbit interaction of light," Phys. Rev. Lett. 104, 253601 (2010).

15. K. Y. Bliokh, E. A. Ostrovskaya, M. A. Alonso, O. G. Rodríguez-Herrera, D. Lara, and C. Dainty, "Spin-to-orbital angular momentum conversion in focusing, scattering, and imaging systems," Opt. Express 19, 26132-26149 (2011).

16. N. B. Baranova, A. Y. Savchenko, and B. Y. Zel'dovich, "Transverse shift of a focal spot due to switching of the sign of circular-polarization," J. Exp. Thoer. Phys. Lett. 59, 232-234 (1994).

17. B. Y. Zel'dovich, N. D. Kundikova, and L. F. Rogacheva, "Observed transverse shift of a focal spot upon a change in the sign of circular polarization," J. Exp. Theor. Phys. Lett. 59, 766-769 (1994).

18. Z. Bomzon, G. Biener, V. Kleiner, and E. Hasman, "Space-variant Pancharatnam-Berry phase optical elements with computer-generated subwavelength gratings," Opt. Lett. 27, 1141-1143 (2002)

19. A. Aiello and J. P. Woerdman, "Role of beam propagation in GoosHänchen and Imbert-Fedorov shifts," Opt. Lett. 33, 1437-1439 (2008).

20. K. Y. Bliokh and A. Aiello, "Goos-Hänchen and Imbert-Fedorov beam shifts: an overview," J. Opt. 15, 014001 (2013).

21. O. Hosten and P. Kwiat, "Observation of the spin Hall effect of light via weak measurements," Science 319, 787-790 (2008).

22. X. Zhou and X. Ling, "Unveiling the photonic spin Hall effect with asymmetric spin-dependent splitting," Opt. Express 24, 3025 (2016).

23. X. Zhou, Z. Xiao, H. Luo, and S. Wen, "Experimental observation of the spin Hall effect of light on a nanometal film via weak measurements," Phys Rev. A 85, 043809 (2012).

24. J. Ren, Y. Li, Y. Qin, R. Wu, J. Yang, Y.-F. Xiao, H. Yang, and Q. Gong, "Spin Hall effect of light reflected from a magnetic thin film," Appl. Phys. Lett. 101, 171103 (2012).

25. K. Bliokh, C. Samlan, C. Prajapati,G. Puentes, N. Viswanathan, F. Nori, "Spin-Hall effect and circular birefringence of a uniaxial crystal plate",
Optica 3, 1039-1047 (2016)

26. B. Z. Rameshti and M. Zareyana, "Charge and spin Hall effect in spin chiral ferromagnetic graphene," Appl. Phys. Lett. 103, 132409 (2013).

27. X. Yin, Z. Ye, J. Rho, Y. Wang, and X. Zhang, "Photonics spin Hall effect at metasurfaces," Science 339, 1405 (2013).

28. Ling, X. Zhou, X. Yi, W. shu, Y. Liu, S. Chen, H. Luo, S. Wen, and D. Fan, "Giant photonic spin Hall effect in momentum space in a structured metamaterial with spatially varying birefringence," Light: Science \& Applications 4, e290 (2015).

29. Y. Liu, Y. Ke, H. Luo, and S. Wen, "Photonic spin Hall effect in metasurfaces: a brief review," Nanophotonics 6, 51 (2017).

30. P. V. Kapitanova, P. Ginzburg, F. J. Rodríguez-Fortuño, D. S. Filonov, P. M. Voroshilov, P. A. Belov, A. N. Poddubny, Y. S. Kivshar, G. A. Wurtz, and A. V. Zayats, "Photonic spin Hall effect in hyperbolic metamaterials for polarization-controlled routing of subwavelength modes, "Nat. Commun. 5, 3226 (2014).

31. X. Zhou, X. Ling, H. Luoa, and S. Wen "Identifying graphene layers via spin Hall effect of light," Appl. Phys. Lett. 101, 251602 (2012).

32. J. Clark and G. Lanzani, "Organic photonics for communications," Nat. Photon. 4, 438 (2010)

33. D.-K. Yang and S.-T. Wu, Fundamentals of Liquid Crystal Devices, 2nd ed. (John Wiley \& Sons, 2015).

34. J.-G. Lim, K. Kwak, and J.-K. Song, "Computation of refractive index and optical retardation in stretched polymer films," Opt. Express 25, 16409 (2017).

35. A. Ciattoni, G. Cincotti, and C. Palma, "Angular momentum dynamics of a paraxial beam in a uniaxial crystal," Phys. Rev. E 67, 036618 (2003)

36. Y. Aharonov, D. Z. Albert, and L. Vaidman, "How the result of a measurement of a component of the spin of a spin-1/2 particle can turn out to be 100," Phys. Rev. Lett. 60, 1351-1354 (1988).

37. I. M. Duck, P. M. Stevenson, and E. C. G. Sudarshan, "The sense in which a 'weak measurement' of a spin- particle's spin component yields a value 100," Phys. Rev. D 40, 2112-2117 (1989).

38. A. G. Kofman, S. Ashhab, and F. Nori, "Nonperturbative theory of weak pre- and post-selected measurements," Phys. Rep. 520, 43-133 (2012)

39. J. Dressel, M. Malik, F. M. Miatto, A. N. Jordan, and R. W. Boyd, "Understanding quantum weak values: basics and applications," Rev. Mod. Phys. 86, 307-316 (2014).

40. N. W. M. Ritchie, J. G. Story, and R. G. Hulet, "Realization of a measurement of a weak value," Phys. Rev. Lett. 66, 1107-1110 (1991).

41. G. Puentes, N. Hermosa, J. P. Torres, "Weak Measurementes with Orbital Angular Momentum Pointer States," Phys Rev. Lett. 109, 040401 (2012)

42. C. Prajapati, S. Pidishety, and N. K. Viswanathan, "Polarimetric measurement method to calculate optical beam shifts," Opt. Lett. 39, 4388-4391 (2014) 